\title{
PRELIMINARY EVALUATION OF NMIS FOR INTERROGATION OF PU AND HEU IN AT400-R CONTAINERS AT MAYAK
}

\author{
T. E. Valentine \\ L. G. Chiang \\ J. T. Mihalczo \\ Oak Ridge National Laboratory \\ P.O. Box 2008 \\ Oak Ridge, Tennessee 37831-6004
}

(423) 574-5612

Date Published: January 2000

\author{
Prepared and operated by \\ OAK RIDGE NATIONAL LABORATORY \\ Oak Ridge, Tennessee 37831-6004 \\ managed by \\ LOCKHEED MARTIN ENERGY RESEARCH CORP. \\ for the \\ U. S. DEPARTMENT OF ENERGY \\ under contract DE-AC05-96OR22464
}




\section{CONTENTS}

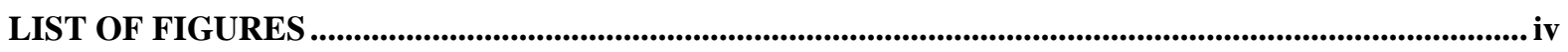

LIST OF TABLES ............................................................................................................................................. iv

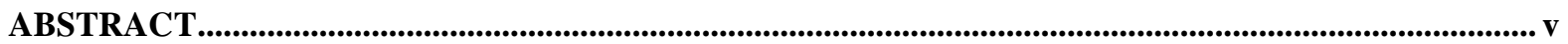

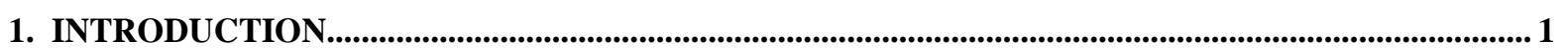

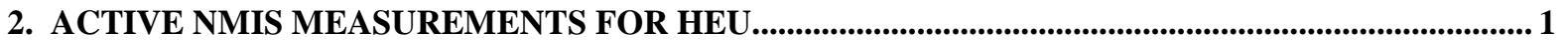

2.1 DEPENDENCE OF NMIS SIGNATURES ON HEU MASS .................................................. 2

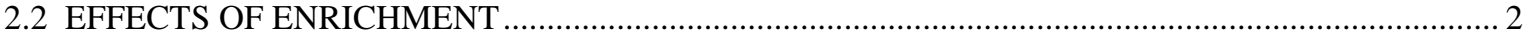

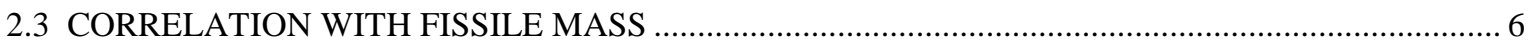

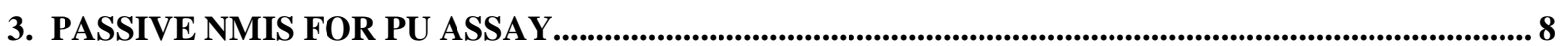

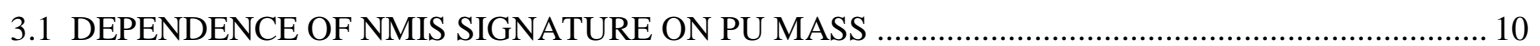

3.2 DETECTOR - AT400R CONTAINER CONFIGURATION .......................................................... 10

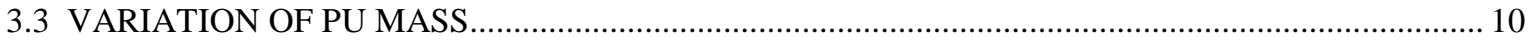

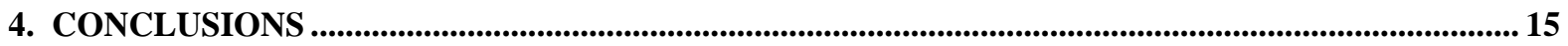

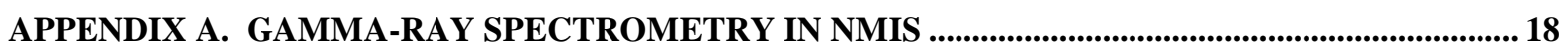




\section{LIST OF FIGURES}

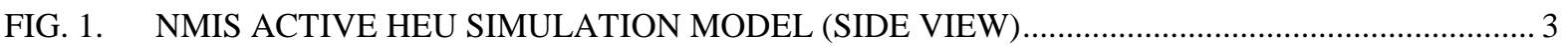

FIG. 2. SIMPLIFIED CONCEPTUAL SKETCH OF CORRELATION BETWEEN A DETECTOR AND SOURCE...

FIG. 3. TIME DISTRIBUTION OF CORRELATED COUNTS AFTER CF FISSION ..............................5

FIG. 4. TIME DISTRIBUTION OF CORRELATED COUNTS PER ${ }^{252}$ CF FISSION FOR VARIOUS

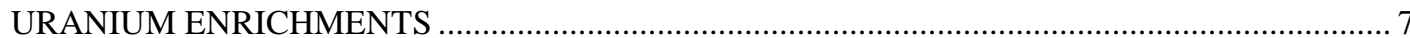

FIG. 5. INDUCED FISSION PORTION OF SIGNATURE DIVIDED BY TRANSMISSION PORTION VS ${ }^{235}$ U MASS FROM ENRICHMENTS VARIATIONS OF 0.2, 36, AND 93 WT\% ${ }^{235} \mathrm{U}$

FIG. 6. PASSIVE NMIS MEASUREMENTS SCALE DIRECTLY WITH SPONTANEOUS FISSION RATE 9

FIG. 7. PASSIVE NMIS SIMULATION MODEL (SIDE VIEW CUT THROUGH CENTER OF PU METAL SPHERE) PASSIVE NMIS SIMULATION MODEL(TOP VIEW CUT THROUGH CENTER OF PU

FIG. 8. PASSIVE NMIS SIM 13

FIG. 9. TIME DISTRIBUTION OF COINCIDENCE COUNTS BETWEEN A PAIR OF DETECTORS ON OPPOSITE SIDES OF THE CONTAINER. 14

FIG. 10. TOTAL COINCIDENT COUNTS VS PU MASS IN AN AT400R CONTAINER. 17

FIG. A-1. NMIS PROCESSOR WITH GAMMA RAY SPECTRUM ANALYZER.

\section{LIST OF TABLES}

TABLE 1. RATIO OF CORRELATED COUNTS DUE TO FISSION TO CORRELATED COUNTS DUE

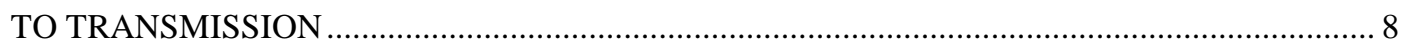




\begin{abstract}
Preliminary Monte Carlo simulations have demonstrated the sensitivity of the NMIS active interrogation method to the amount of uranium fissile material stored in AT400-R containers that are being proposed for use in the Mayak facility. Properties of the time-of-flight signature can be used to determine the absence of one of the uranium metal spheres or to determine if a different enrichment sphere is present in the container. The tail of the time-offlight signature from induced fission is directly dependent on the amount of uranium 235 present in the containers, and a particular ratio of the correlated counts due to fission to the correlated counts due to transmission is nearly linear with ${ }^{235} \mathrm{U}$ mass. These simulations demonstrate that the NMIS active method can be used to assay the amount of ${ }^{235} \mathrm{U}$ with a sensitivity of coefficient of $\sim 0.1$ per kg ${ }^{235} \mathrm{U}$ (approximately $10 \%$ change in the ratio per kilogram of $\left.{ }^{235} \mathrm{U}\right)$. These calculations have shown that these active measurements with a ${ }^{252} \mathrm{Cf}$ source of $1 \times 10^{6}$ fission per sec would require the order of a few minutes of data accumulation time for a container with two $8 \mathrm{~kg}$ spheres, and since NMIS operates in real time, 1.6 minutes of measurement time is required. This measurement time is short.

The calculations for $\mathrm{Pu}$ have shown that NMIS in the passive mode (no $\mathrm{Cf}$ source) can determine the mass of $\mathrm{Pu}$ in AT400-R containers with short measurement times of a few minutes. The sensitivity of the proposed detectors to gamma rays should enhance this measurement method since the gamma rays from fission, induced or spontaneous, escape the container more easily than neutrons. In addition to the time correlation measurements, the multiplicity options of NMIS allow conventional multiplicity measurements that, depending on the type of detector, can include prompt gamma rays from fission.
\end{abstract}

If gamma ray spectrometry is included in the NMIS processor, then one NMIS system can perform the desired NMC\&A measurements for use at Mayak (i.e., HEU, Pu, and $\mathrm{Pu}$ isotopics). Higher order NMIS correlation measurements have promise for determining the $\mathrm{Pu}$ shape passively also if desired, but this requires four detectors. Active higher order measurements with $\mathrm{Pu}$ can separate out the effects of induced fission and spontaneous fission and thus may yield information on the ratio of ${ }^{239} \mathrm{Pu}$ to ${ }^{240} \mathrm{Pu}$. Incorporation of gamma-ray spectrometry is discussed in Appendix A.

These capabilities should be verified by measurements both with uranium and plutonium. 


\section{INTRODUCTION}

This report summaries a preliminary assessment of the use of the Nuclear Material Identification System (NMIS) for nondestructive assay of the content of AT400-R storage containers used at the Mayak storage facility. This assessment evaluates the use of NMIS in the active mode for use with highly enriched uranium (HEU) metal and in the passive mode (no Cf source) for plutonium metal. The primary tool for this assessment is a combined neutron-gamma ray transport code MCNP-DSP (Refs. $1 \& 2$ ) which calculates directly all the signatures measured by the NMIS in both passive and active modes. These calculations are routinely used for planning and interpreting measurements. The use of the same NMIS measurement system for both uranium and plutonium would be cost effective. A description of the active NMIS as applied to enriched uranium metal spheres is given in Sect. 2 and that for $\mathrm{Pu}$ metal spheres in Sect. 3 and shows how the signatures depend on fissile mass. Conclusions are presented in Sect. 4. An Appendix to this report describes how gamma-ray spectrometry has been incorporated into this NMIS processor which would allow another desired measurement in this one system (Pu isotopics) and increase cost effectiveness further. In recent measurements with hundreds of birdcages at the Oak Ridge Y-12 Plant over several months the enrichment and uranium mass of HEU metal has been measured in situ to within a total variation of $\pm 5 \%$ (3 sigma).

This work was suggested by D. Phillipone of Bechtel Corp. and was supported by ORNL program development funding.

\section{ACTIVE NMIS MEASUREMENTS FOR HEU}

The ${ }^{252} \mathrm{Cf}$ source was located on the axis of the container at the bottom to minimize the amount of hydrogenous material the ${ }^{252} \mathrm{Cf}$ neutrons had to penetrate to reach the fissile material. This choice was made to reduce CPU time. It is probably also practical to measure through the sides of the container at each sphere height but this was not calculated. The detectors selected for these simulations are fast plastic BC420 scintillation detectors that are sensitive to both neutrons and gamma rays. The detectors were located on the top of the container. The outer diameter of the detector on the axis of the container was determined such that the detector was completely within the solid angle defined between the source and the outer diameter of the upper sphere. The outside diameter of the plastic scintillator was $15.64 \mathrm{~cm}$. This source-detector configuration results in the active NMIS measurement being a time-of-flight transmission measurement through both spheres. An additional detector was located adjacent to the first such that its center was at an effective radius of $15.64 \mathrm{~cm}$ (i.e., its axis was displaced $15.64 \mathrm{~cm}$ from the axis of the container). If the lower sphere were not in the container, then there would be more particles passing directly from the source to this detector. The use of an associated particle sealed neutron generator (ABSTNG) that produces $14 \mathrm{MeV}$ neutrons that would penetrate to the HEU much easier than ${ }^{252} \mathrm{Cf}$ neutrons would reach the desired statistical accuracy faster but adds the complication of ABSTNG and its power supply. 
A sketch of the Monte Carlo model of the container is given in Fig. 1. Coupled neutrongamma ray calculations were performed for 100 million ${ }^{252} \mathrm{Cf}$ source fissions. A typical ${ }^{252} \mathrm{Cf}$ source for an active measurement could easily be 1 million fissions per second, so this calculation corresponds to less than 1.6 minutes of measurement time. To investigate the sensitivity to fissile content, a variety of calculations were performed. The ${ }^{235} \mathrm{U}$ enrichment was varied $\left(0.2,36\right.$, and $\left.93.2 \mathrm{wt} \%{ }^{235} \mathrm{U}\right)$ to investigate sensitivity to fissile mass. It was assumed that the uranium density was $18.75 \mathrm{~g} / \mathrm{cm}^{3}$ for the $8-\mathrm{kg}$ spheres. The variations of these models include no spheres, the lower sphere only, the upper sphere only, and both spheres.

\subsection{DEPENDENCE OF NMIS SIGNATURES ON HEU MASS}

Because all correlated counts from induced fission are related to the fissile mass of HEU, many of the NWIS signatures depend on HEU mass. A sketch of the time distribution of counts after Cf fission is given in Fig. 2 which shows the various contributions to the signal: transmitted gamma rays, transmitted neutrons, scattered neutrons and gamma rays, and neutrons and gamma rays from fission. NMIS also acquires delayed multiplicities, that is, multiplicity counting with a time window triggered by $\mathrm{Cf}$ fission that can be delayed until after transmitted gamma rays and neutrons from $\mathrm{Cf}$ fission have reached the detector. It also acquires conventional multiplicities with detection event and randomly triggered time windows. Correlations between detectors also depend on HEU mass. The signature that requires the least CPU time to calculate is the time distribution of correlated neutrons and gamma rays after $\mathrm{Cf}$ fission. Thus, it was used in this evaluation. To correct somewhat for transmission through the container material, the ratio of the correlated counts from the induced fission part of the correlation ( $\mathrm{Cf}$ neutrons have to get in and induce fission and neutron and gamma rays have to get out) to the transmitted neutrons and scattered neutrons and gamma rays ( $\mathrm{Cf}$ fission particles have to get in and get out to the detector) was used as the calculated parameter in this evaluation that shows the mass dependence. Delayed multiplicity counting and many other signatures would also be useful and all signatures are obtained simultaneously by NMIS in a measurement, but were not calculated in these simulations.

\subsection{EFFECTS OF ENRICHMENT}

This active pulsed neutron method is highly sensitive to the amount of fissile material in the container. The correlated counts per ${ }^{252} \mathrm{Cf}$ fission as a function of time after ${ }^{252} \mathrm{Cf}$ fission (time-of-flight) are given in Fig. 3 for simulations with $93.15 \mathrm{wt} \%, 8$-kg HEU spheres in the AT400-R container. Also shown are the calculated results for no spheres in the container. Several observations can be made about these time-of-flight signatures. First, the direct gamma ray transmission (occurring after the flight time of gamma rays from the source to the detector, $\sim 1.6 \mathrm{nsec}$. The scattered gamma-ray portion of the signature occurs at about $5 \mathrm{nsec}$. The arrival time for $10 \mathrm{MeV}$ neutrons from the source occurs at $11.5 \mathrm{nsec}$. The portion of the signature between $11.5 \mathrm{nsec}$ and $37.5 \mathrm{nsec}$ is primarily due to transmitted neutrons although a small portion of the response in this region is due to fission. A crossover point is indicated on the figure at a time of $\sim 38 \mathrm{nsec}$. At this point the response with no spheres in the 


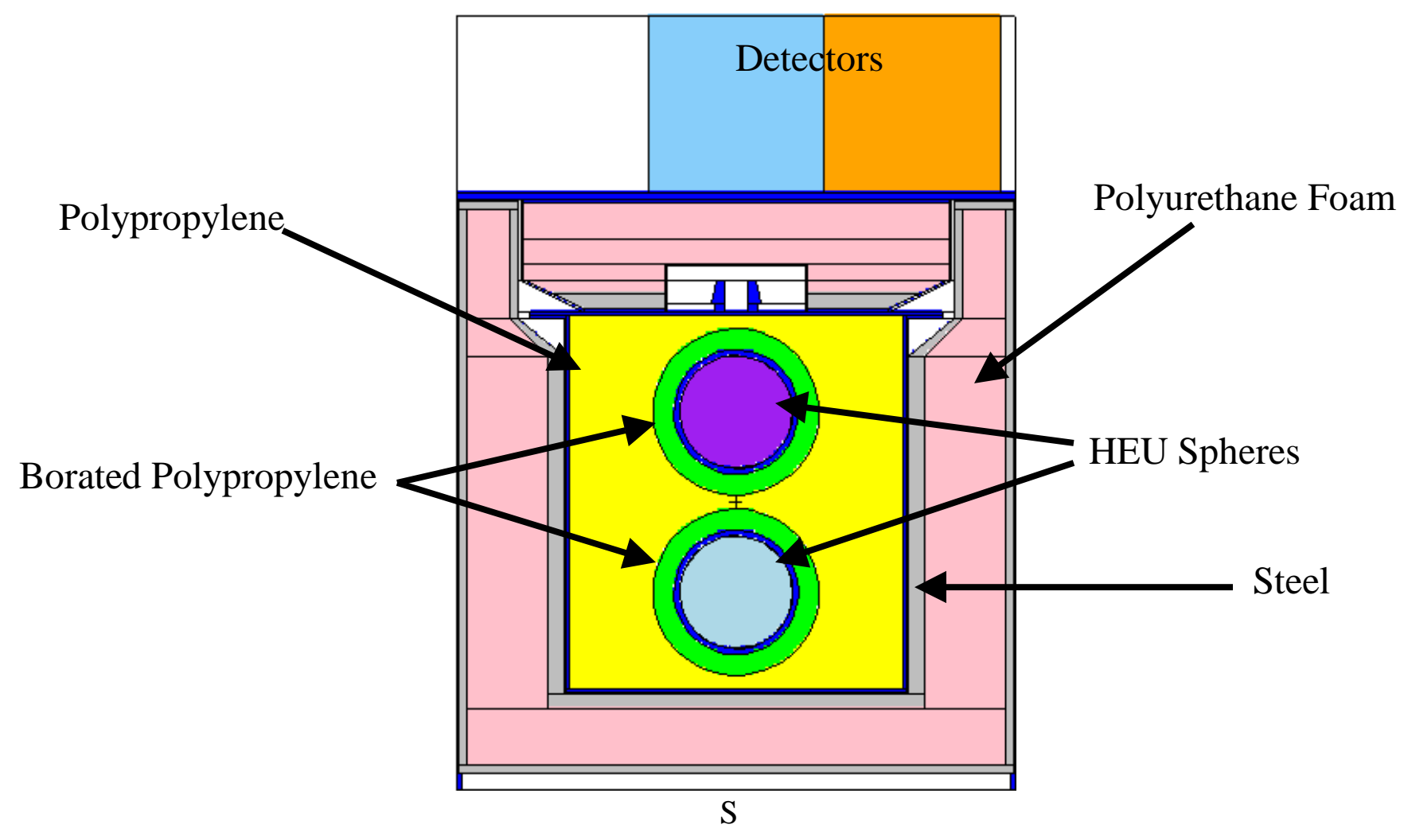

Fig. 1. NMIS active HEU simulation model (side view). 


\section{THE AVERAGE CORRELATED TIME ANALYSIS SIGNATURE CONSISTS}

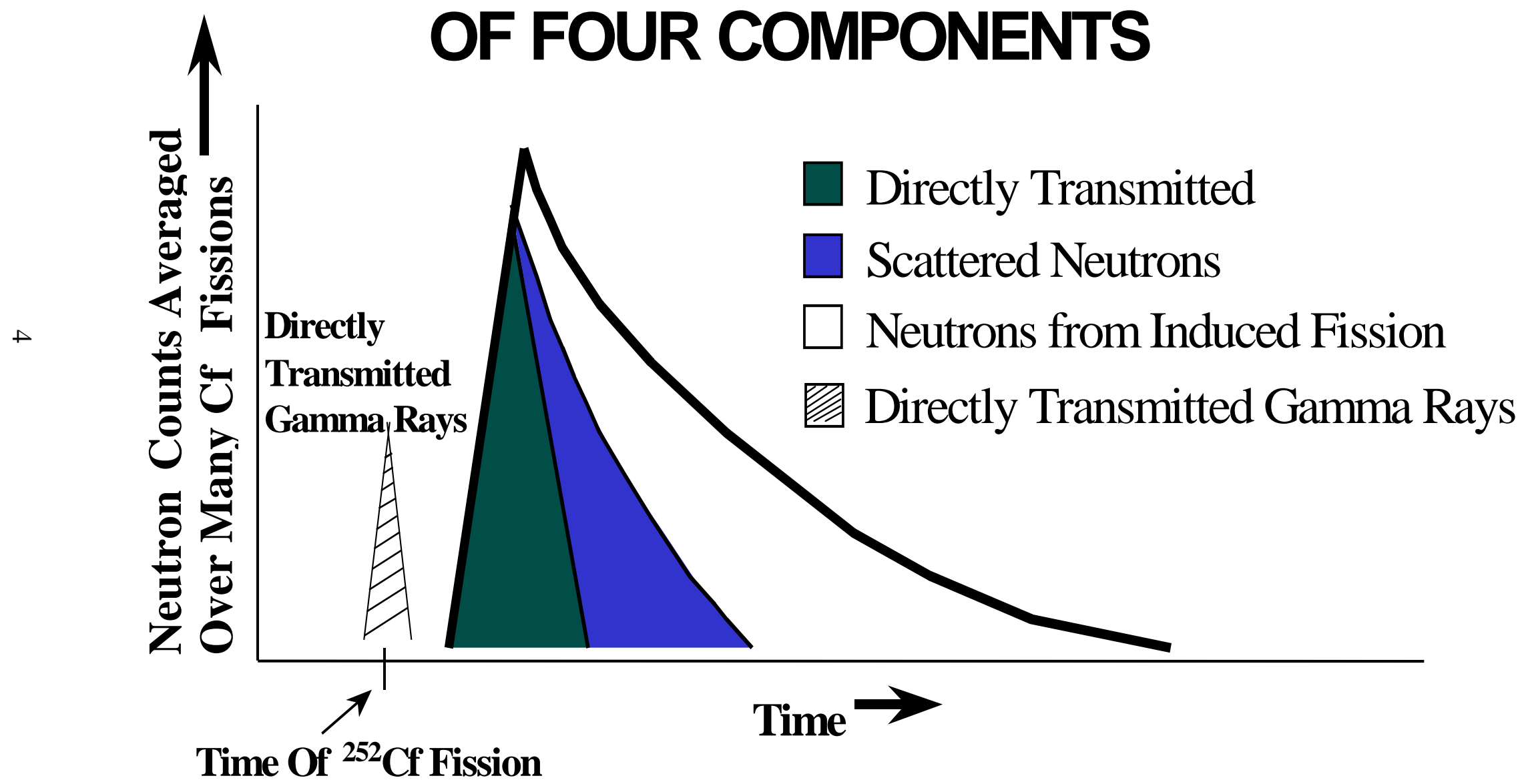

Fig. 2. Simplified conceptual sketch of correlation between a detector and source. 


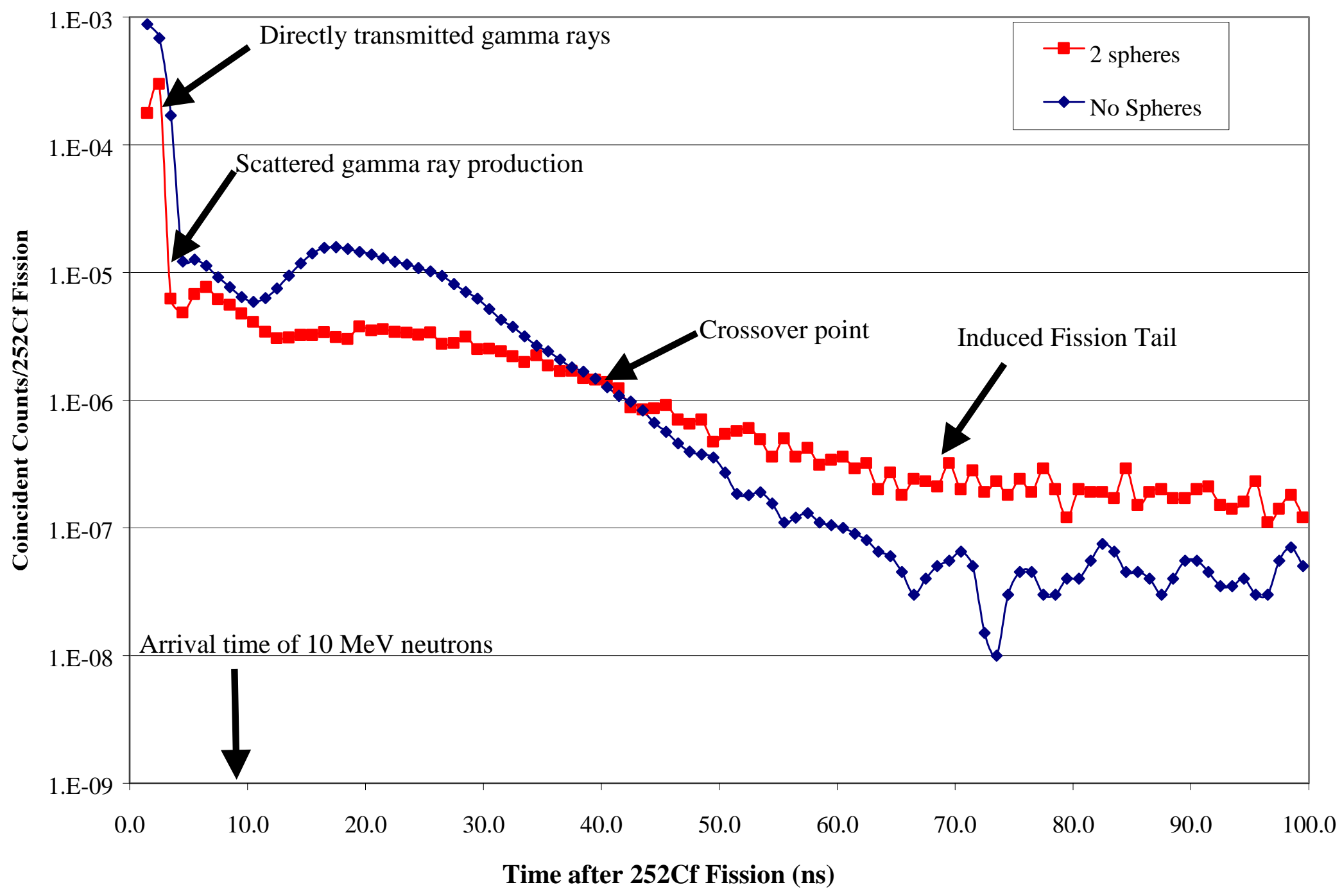

Fig. 3. Time distribution correlated counts after ${ }^{252} \mathrm{Cf}$ fission. 
container is the same as that with spheres present. For times past the crossover point, the response for no spheres present is lower than that with the spheres present (either one or both). This higher signal at longer times come primarily from induced fission. Clearly, the signals with spheres present are much higher than without between 40 and $100 \mathrm{nsec}$ because of induced fission chain multiplication. The signals at latter times are not due to lower energy neutrons because the thresholds of the plastic scintillation detectors are set to not detect neutrons much below $1.5 \mathrm{MeV}$. Thus, this response at long time is primarily due to higher energy neutrons from induced fission. The time channels are $1 \mathrm{~ns}$ and a value of $10^{-6}$ counts per $\mathrm{Cf}$ fissions means 100 counts in one channel in $100 \mathrm{sec}$ for a ${ }^{252} \mathrm{Cf}$ source of $10^{6}$ fissions per second. Integration over all channels gives thousands of counts from induced fission. The tail of the time-of-flight signature is highly dependent on the total fissile mass. It is affected by the uranium enrichment. The time-of-flight signatures between the source and the detector located on the axis of the container for the three different enriched 8-kg uranium metal spheres are given in Fig. 4. The enrichment variation was used only to explore sensitivity to ${ }^{235} \mathrm{U}$. The most significant difference between the three time-of-flight signatures occurs in the region of the induced fission tail. More correlated counts are obtained with the $93 \%{ }^{235} \mathrm{U}$ enriched metal sphere because more ${ }^{235} \mathrm{U}$ is present in this

sphere. As the amount of ${ }^{235} \mathrm{U}$ is reduced, the number of correlated counts in the region of the induced fission tail decreases.

\subsection{CORRELATION WITH FISSILE MASS}

The portion of the time-of-flight signatures that is directly related to the fissile mass can be used to determine the mass of ${ }^{235} \mathrm{U}$ present. One method to analyze these particular time-offlight signatures is to calculate the ratio of the integral of the correlated counts past the crossover time to the integral of the correlated counts between the arrival time of $10-\mathrm{MeV}$ neutrons and the crossover time. Attenuation effects are of concern in evaluating these signatures. Using this ratio is an attempt in some approximation to remove attenuation effects. The attenuation effects in the numerator and denominator of this ratio are the same to first order and thus tend to cancel. For both the numerator and denominator the source neutrons have to penetrate into the fissile material. Both the transmitted and induced fission neutrons also have to emerge and reach the detector. 


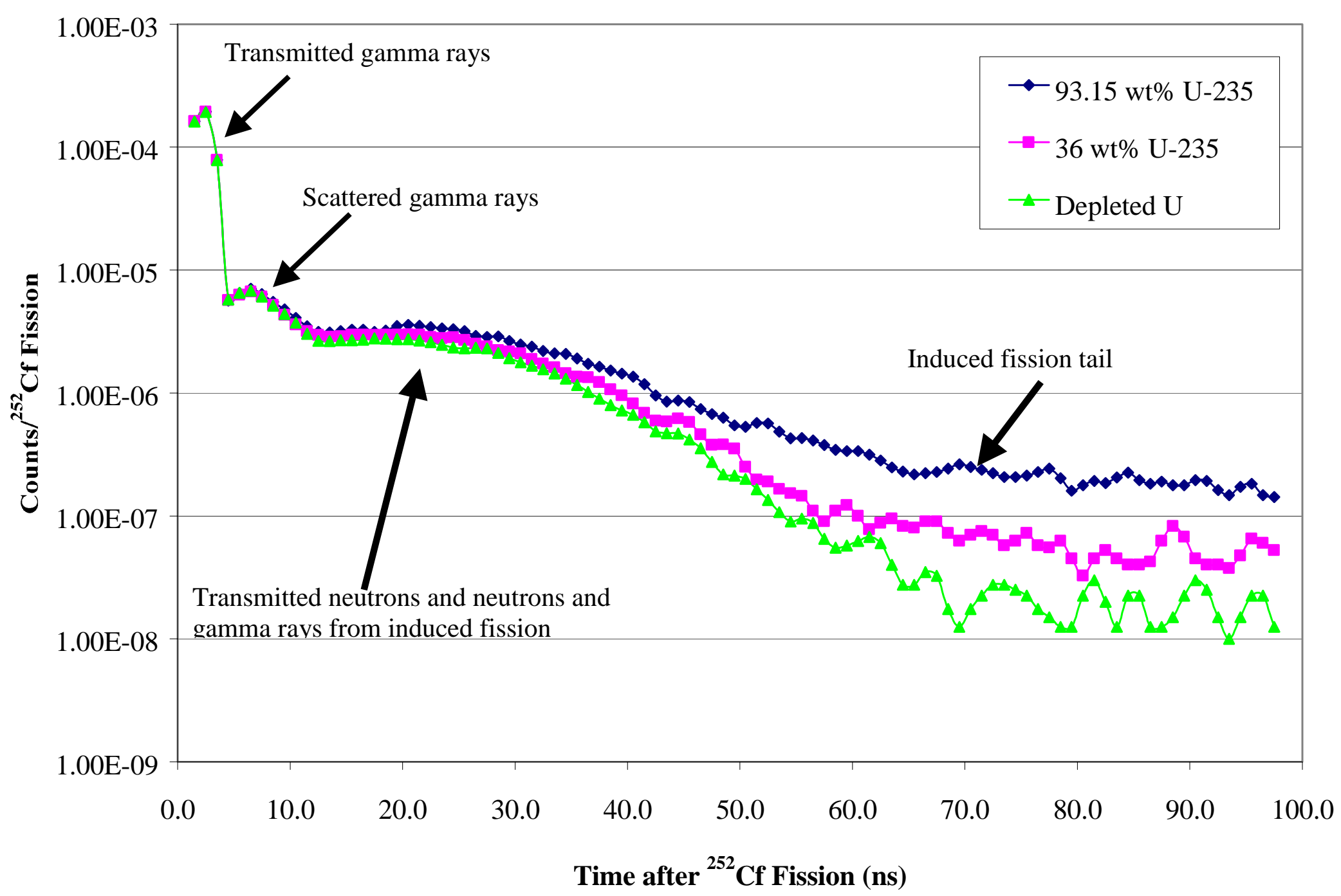

Fig. 4. Time distribution of correlated counts per ${ }^{252} \mathrm{Cf}$ fission for various uranium enrichments. 
These ratios for the different enrichments of the $8-\mathrm{kg}$ uranium metal spheres are presented in Table 1. This ratio exhibits a near linear trend as a function of the ${ }^{235} \mathrm{U}$ mass as shown in Fig. 5. Deviations from linear is due to multiplication. The sensitivity of this ratio is approximately 0.06 per $\mathrm{kg}$ of ${ }^{235} \mathrm{U}$. The sensitivity coefficient is defined as $\Delta R / \Delta m / \bar{R}$ where $\mathrm{m}$ is the uranium mass in kilograms and $\bar{R}$ is the average ratio as a function of mass. Thus, active NMIS measurements can be used to verify the fissile mass of spheres of HEU in the AT400-R container. Once a measurement is performed, it can be used as a template whose features at long times are related to fissile mass or it could be used as a calibration. For less accurate mass determination a calculated calibration could be used.

Table 1. Ratio of correlated counts due to fission to correlated counts due to transmission

\begin{tabular}{|c|c|}
\hline Total ${ }^{235} \mathrm{U}$ mass $(\mathrm{kg})$ & Ratio \\
\hline \multicolumn{2}{|c|}{ Both spheres } \\
\hline $14.90\left(93.15 \mathrm{wt} \%, 8 \_\mathrm{kg}\right)$ & 0.385 \\
\hline $5.76(36 \mathrm{wt} \%, 8-\mathrm{kg})$ & 0.211 \\
\hline $0.03(0.2 \mathrm{wt} \%, 8-\mathrm{kg})$ & 0.152 \\
\hline \multicolumn{2}{|c|}{ Upper sphere only } \\
\hline $7.45(93.15 \mathrm{wt} \%, 8-\mathrm{kg})$ & 0.210 \\
\hline $2.88(36 \mathrm{wt} \%, 8-\mathrm{kg})$ & 0.131 \\
\hline $0.02(0.2 \mathrm{wt} \%, 8-\mathrm{kg})$ & 0.104 \\
\hline
\end{tabular}

Essentially, this ratio varies linearly with fissile mass and can be used to determine the appropriate fissile mass. Many other NMIS signatures including source, detection event, and randomly triggered multiplicities can also be used to obtain fissile mass. In an application all signatures dependent on mass will be evaluated and certainly more than one detector will be used.

\section{PASSIVE NMIS FOR PU ASSAY}

NMIS in the passive mode has been shown to be very sensitive to Pu mass in blind tests for DSWA at Los Alamos National Laboratory (LANL) in August 1997 and for template measurements at Pantex in December 1997. NMIS correlations for passive measurements depend on the ${ }^{240} \mathrm{Pu}$ content and any induced fission from these inherent source neutrons. Of course, the passive results depend on spontaneous fission of ${ }^{240} \mathrm{Pu}$ and induced fission of ${ }^{239} \mathrm{Pu}$. To obtain the ${ }^{239} \mathrm{Pu}$ mass, the ratio of ${ }^{239} \mathrm{Pu}$ to ${ }^{240} \mathrm{Pu}$ must be known. Calculations for a variety of $\mathrm{Pu}$ masses were performed to investigate sensitivity of the measured signatures to fissile mass. A measured calibration or a calculated calibration standard could be used to determine the $\mathrm{Pu}$ mass assuming isotopic compositions are known. Again, only one signature was utilized in these simulations, the cross correlation between detectors. 


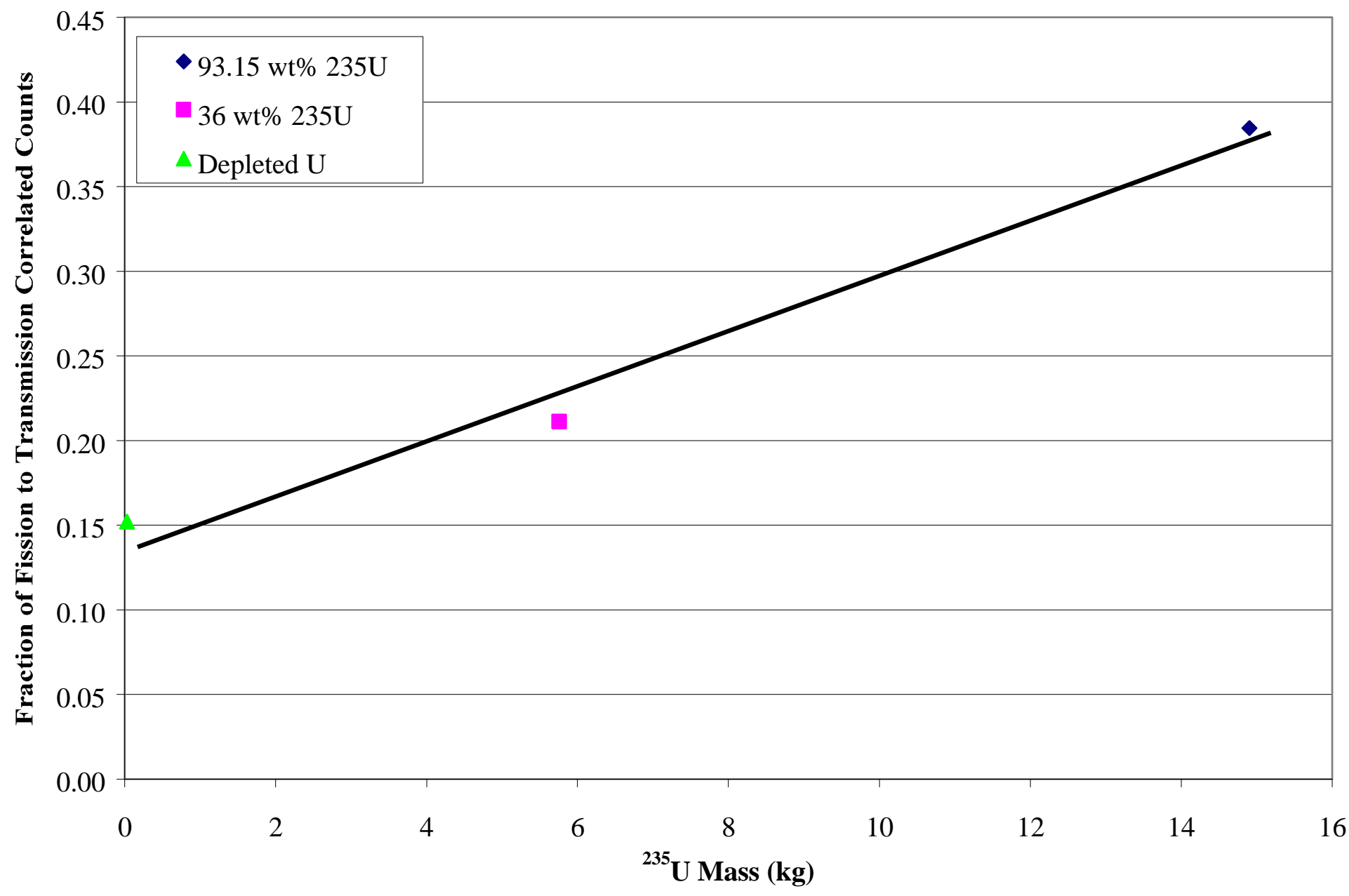

Fig. 5. Induced fission portion of signature divided by transmission portion vs ${ }^{235} \mathrm{U}$ mass from enrichments variations of $0.2,36$, and $93 \mathrm{wt} \%{ }^{235} \mathrm{U}$. 


\subsection{DEPENDENCE OF NMIS SIGNATURE ON PU MASS}

Passive correlation counting signatures from NMIS can be used for Pu mass determination. The time distribution of counts in one detector after a count in another detector is measured. For second order correlations this is the temporal distribution of doubles (third order-triples, etc.). The integral of correlated time distribution of a second order signature gives the number of doubles in a multiplicity measurement. So in a sense, multiplicity counting is a subset of the correlation functions (i.e. their integrals). Obviously, the correlation functions have more information than their integrals. NMIS also does passive multiplicity counting in the same way a commercial multiplicity counter with both event triggered and randomly triggered time windows, but the data is processed like as in $1 \mathrm{GHz}$ shift register. Since the time window is much shorter than conventional multiplicity counters (512 nsec vs $64 \mu \mathrm{sec}$ ), the number of accidental coincidence may be much less than for conventional multiplicity counting as it has been used for years for Pu mass determinations.

Many measurements using $\mathrm{Cf}$ sources as a surrogate for $\mathrm{Pu}$ have shown that the correlation function (temporal distribution of doubles) depends on spontaneous fission rate. One such plot is given in Fig. 6 where the $\mathrm{Cf}$ source intensity was increased in four steps. The signatures depend on source fission rate or mass. The same dependency has been shown for $\mathrm{Pu}$ mass in pits. In this analysis the cross correlation between a pair of the detectors was used to illustrate the dependence on Pu mass.

\subsection{DETECTOR - AT400R CONTAINER CONFIGURATION}

The configuration of the AT400-R container (side view) used in these calculations is given in Fig. 7. A top view of the calculation model with the detectors in place is shown in Fig. 8. The $\mathrm{Pu}$ spheres were centered in the $10.38-\mathrm{cm}$ diameter spherical cavities and held in position by an Al hemispherical shell of thickness to center the Pu spheres. The AT400-R container was surrounded by four large plastic scintillation detectors that essentially formed a box with inside dimensions of $50 \mathrm{~cm}$. Such large detectors have been used at ORNL for other measurements. This arrangement surrounds the container on all sides with plastic scintillation detectors. Actually smaller detectors could be used and previous NMIS measurements with Pu parts in ALR8 containers and one part in an AT400 container used $6 \times 6 \times 4$-in.-thick plastic scintillators.

\subsection{VARIATION OF PU MASS}

To investigate the sensitivity to Pu mass five calculations were performed for spheres of 2, 1.5 , and 1.0 and $0.5 \mathrm{~kg}$ of delta phase Pu with $1 \mathrm{wt} \%$ gallium and $6 \mathrm{wt} \%{ }^{240} \mathrm{Pu}$. In one calculation, sphere masses were mixed (i.e., one $2-\mathrm{kg}$ and one $1.5-\mathrm{kg}$ sphere). These variations of $\mathrm{Pu}$ mass were evaluated to establish sensitivity to $\mathrm{Pu}$ mass. The covariance functions between a pair of these large plastic scintillator detectors on opposite sides of the container for all cases are plotted in Fig. 9. These covariance functions are the temporal 


\section{PASSIVE NMIS MEASUREMENTS SCALE DIRECTLY WITH SPONTANEOUS FISSION RATE}

- Passive NMIS measurements of four Cf-252 spontaneous fission sources of nearly identical mass

Passive Coincidence Distribution

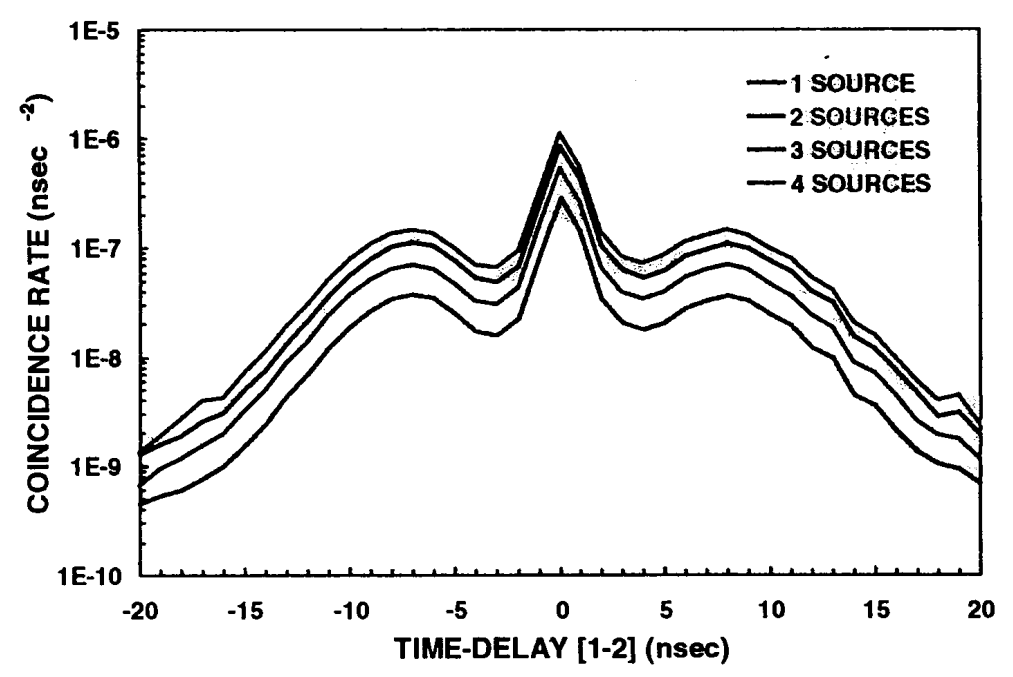

Area Under Distribution vs

Cf-252 mass

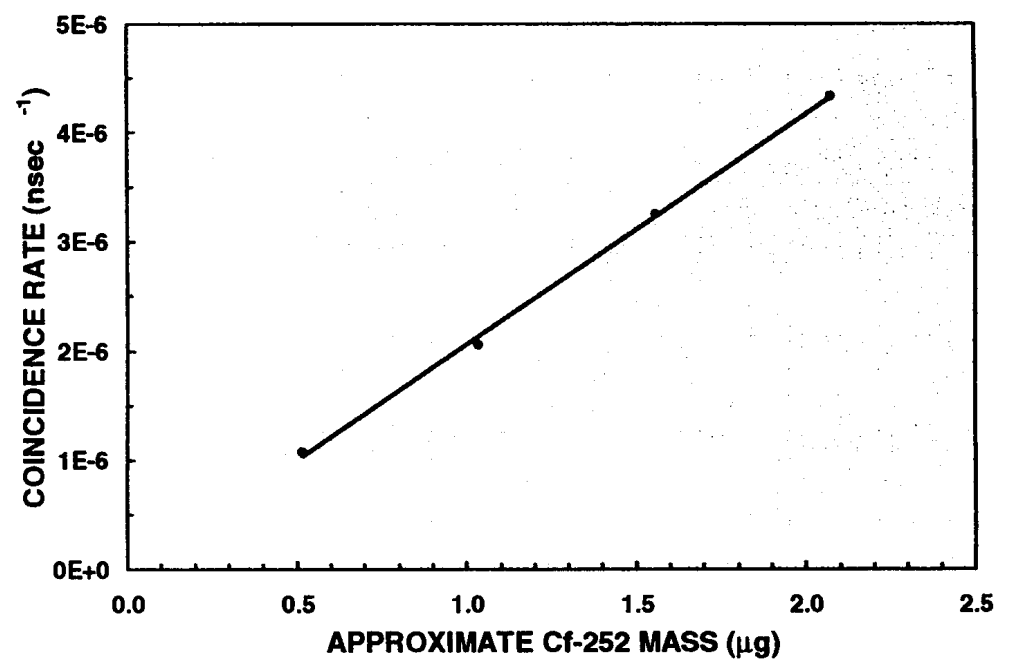

Fig. 6. Passive NMIS measurements scale directly with spontaneous fission rate. 


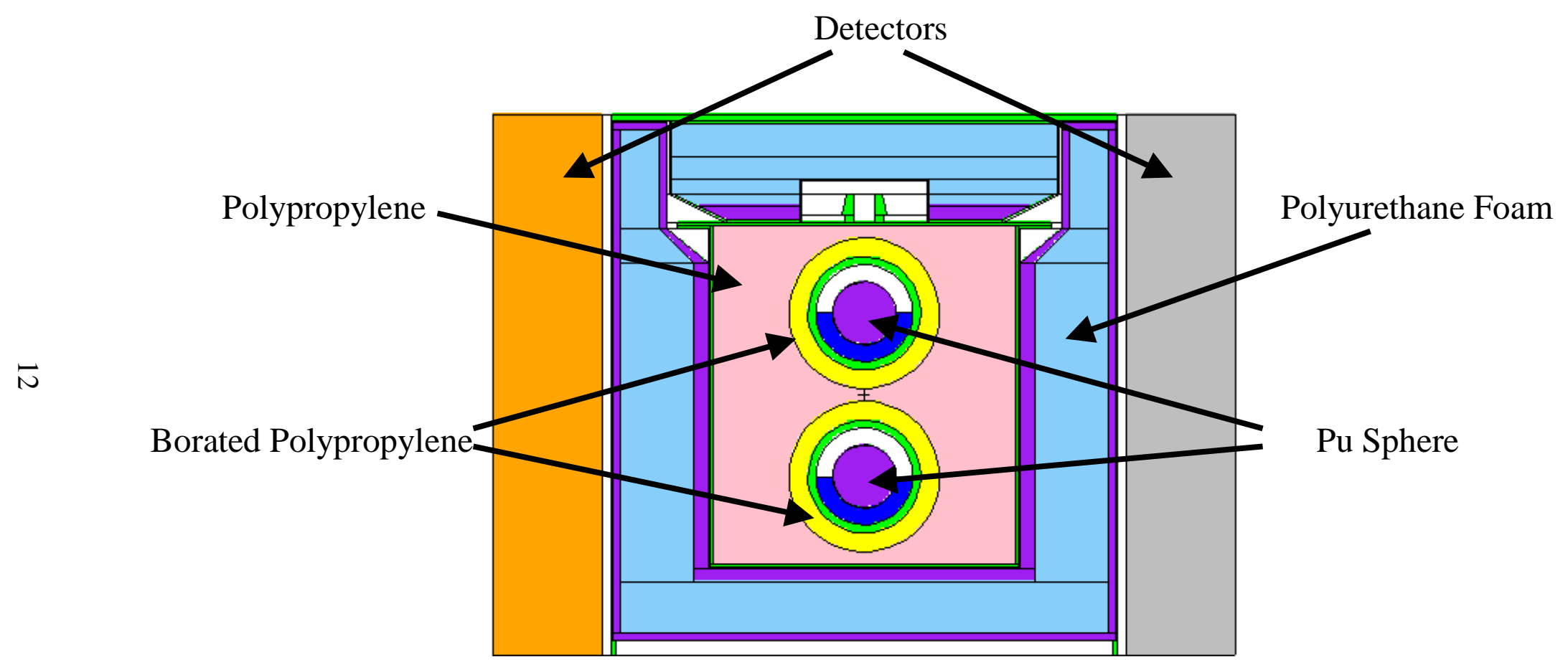

Fig. 7. Passive NMIS simulation model (side view cut through center of Pu metal sphere). 


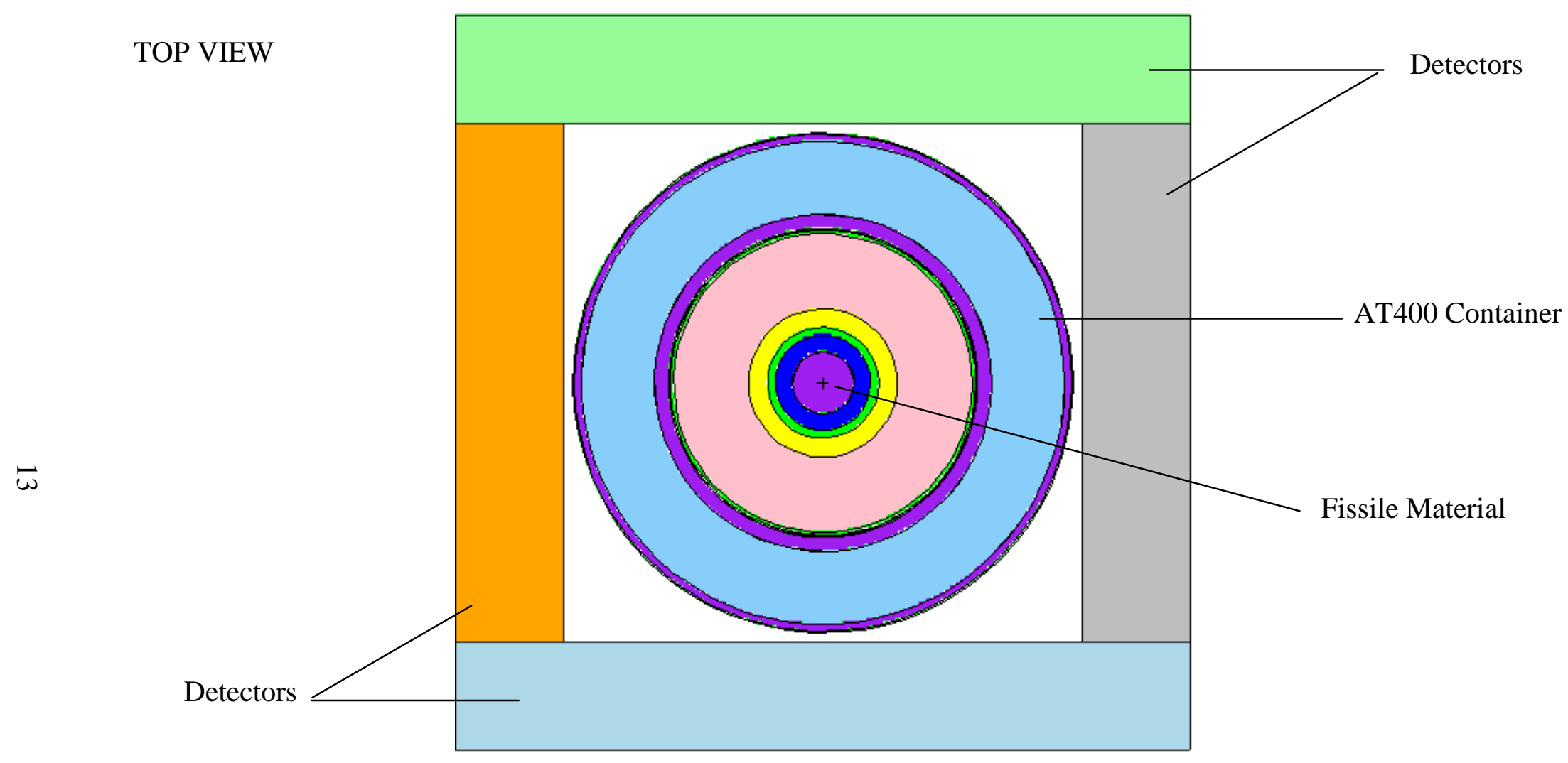

Fig. 8. Passive NMIS simulation model (top view cut through center of Pu metal sphere). 


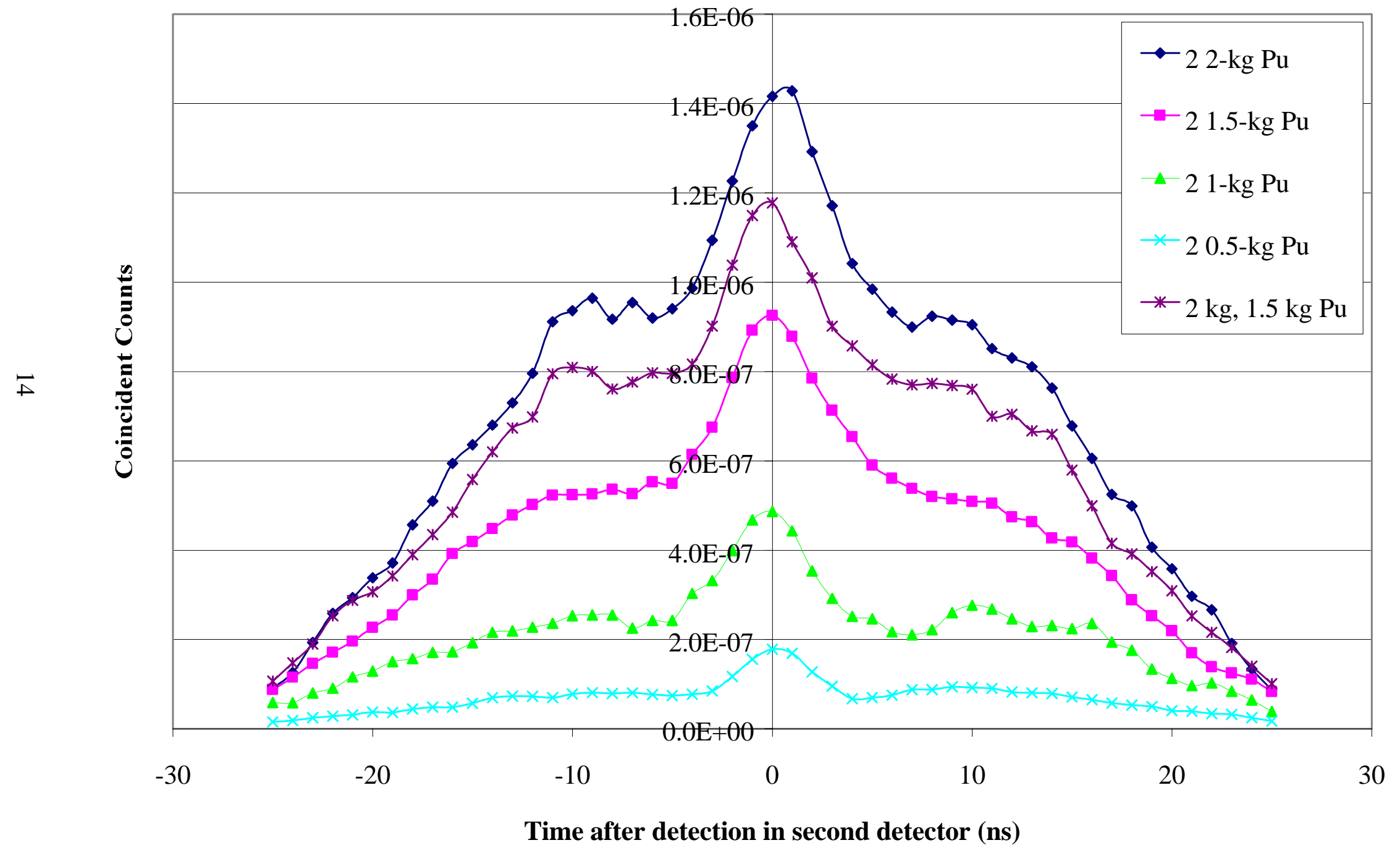

Fig. 9. Time distribution of coincidence counts between a pair of detectors on opposite sides of the container. 
distributions of coincident counts (doublets) and are proportional to Pu mass. The coincident gamma-ray signatures are primarily at time 0, (i.e. two gamma rays from fission each reach the detectors with small flight time differences). The integrals of these covariance functions are the total number of coincidence counts and are plotted as a function of $\mathrm{Pu}$ mass in Fig. 10. These calculations represent 900000 spontaneous fission events of ${ }^{240} \mathrm{Pu}$ and thus would correspond to a measurement time of $\sim 1.0 \mathrm{~min}$. for the $2-\mathrm{kg}$ spheres. For the case of $2 \mathrm{~kg}$ spheres, the doublet count rate was $\sim 4,000$ per minute so 10,000 doubles could be obtained in a few minutes. Of course to obtain $\mathrm{Pu}$ mass, $\mathrm{Pu}$ isotopics are required. Again many other NMIS signatures such as multiplicities could also be utilized but were not evaluated in this study. Recent NMIS measurements with fission sources have shown that NMIS signatures can be used for fissile shape determination (Ref. 3, 4, and 5).

\section{CONCLUSIONS}

These Monte Carlo simulations have demonstrated the sensitivity of the NMIS active interrogation method to the amount of uranium fissile material stored in AT400-R containers that are being proposed for use in the Mayak facility. Properties of the time-of-flight signature can be used to determine the absence of one of the uranium metal spheres or to determine if a different enrichment sphere is present in the container. The tail of the time-offlight signature from induced fission is directly dependent on the amount of uranium 235 present in the containers, and a particular ratio of the correlated counts due to fission to the correlated counts due to transmission is nearly linear with ${ }^{235} \mathrm{U}$ mass. Other NMIS signatures including multiplicity measurements with source, detection event, and randomly triggered time windows would be used. These simulations demonstrate that the NMIS active method can be used to assay the amount of ${ }^{235} \mathrm{U}$ with a sensitivity of coefficient of $\sim 0.1$ per kg ${ }^{235} \mathrm{U}$ (approximately $10 \%$ change in the ratio per kilogram of ${ }^{235} \mathrm{U}$ ). Other time correlation signatures can have a higher sensitivity to HEU mass. These calculations have shown that these active measurements with a ${ }^{252} \mathrm{Cf}$ source of $1 \times 10^{6}$ fission per sec would require the order of a few minutes of data accumulation time for a container with two $8 \mathrm{~kg}$ spheres, and since NMIS operates in real time, a few minutes measurement time is required. This measurement time is short.

The calculations for $\mathrm{Pu}$ have shown that NMIS in the passive mode (no $\mathrm{Cf}$ source) can determine the mass of $\mathrm{Pu}$ in AT400-R containers with short measurement times (a few minutes) with large plastic scintillation detectors around the container. Higher order NMIS correlation measurements can determine $\mathrm{Pu}$ shape passively also if desired, but this requires four detectors.

NMIS has physics advantages over other methods since prompt gamma rays from fission more readily escape the container and can be detected. Recent measurements at the Oak Ridge Y-12 Plant have shown that the stability of the detection system proposed was adequate to determine the uranium mass and enrichment of HEU metal and hundreds of birdcages over a several month measurement period to within $\pm 5 \%$ ( 3 sigma). 
If gamma-ray spectrometry is included in the NMIS processor, then one NMIS system can perform the desired NMC\&A measurements for use at Mayak (i.e., HEU, Pu, and Pu isotopics). . Incorporation of gamma-ray spectrometry is discussed in Appendix A. These capabilities should be verified by measurements. 


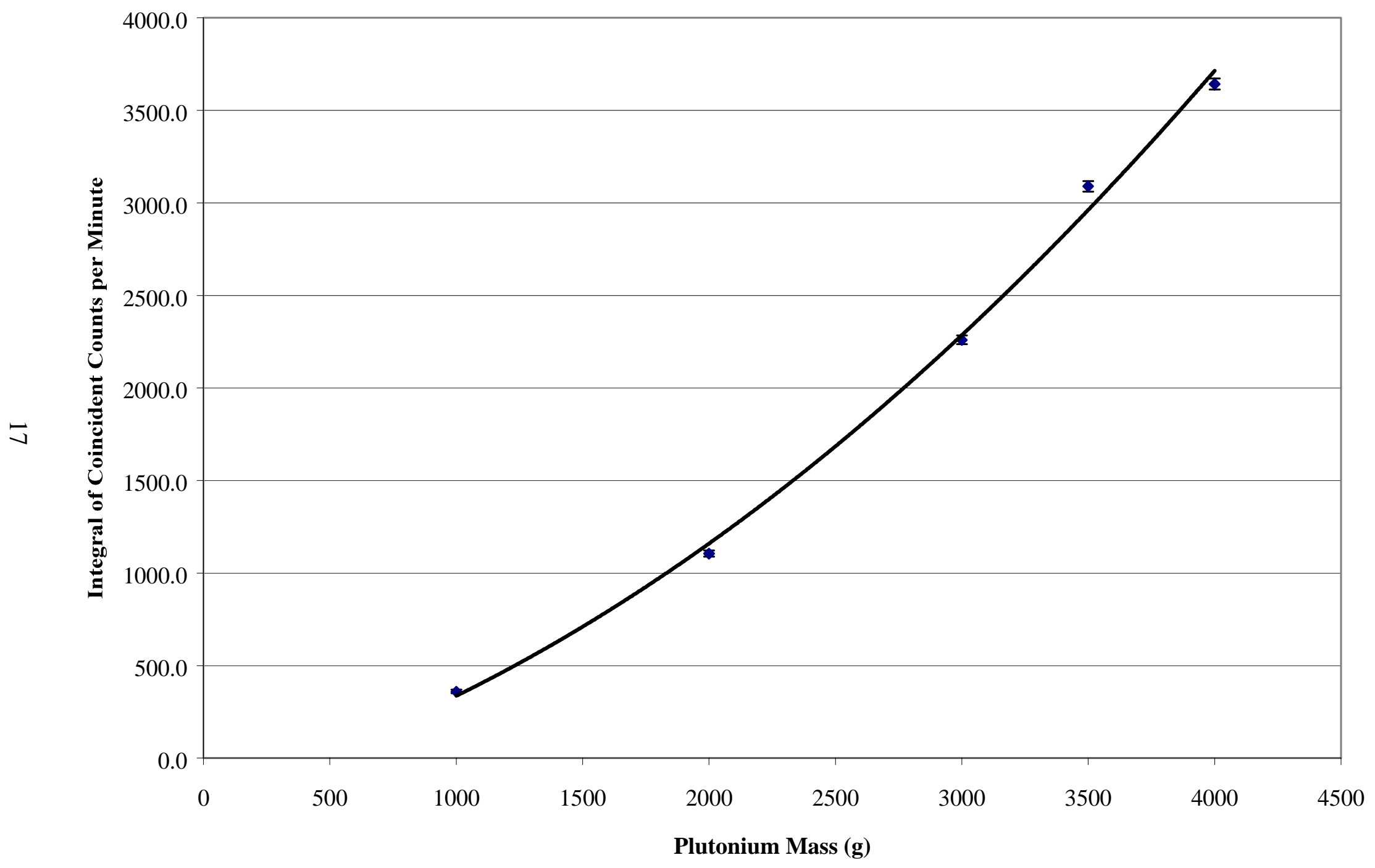

Fig. 10. Total coincident counts vs Pu mass in an AT400R container. 


\section{APPENDIX A. GAMMA-RAY SPECTROMETRY IN NMIS}

A gamma-ray spectrometry processor board (ORTEC Trump 8K) has been incorporated into a NMIS processor as shown in Fig. A-1. Tests have been performed with the NMIS and the multichannel analyzer (MCA) board operating simultaneously without any significant reduction in the processing time for either. This results from the fact that once the MCA data acquisition has started, it does not require much CPU time which is fully occupied with the usual NMIS processing. The incorporation of this gamma-ray spectrometry allows the measurement of HEU and Pu mass and isotopics all in the same box. 


\section{NMIS Processor with Gamma Ray Spectrum Analyzer}

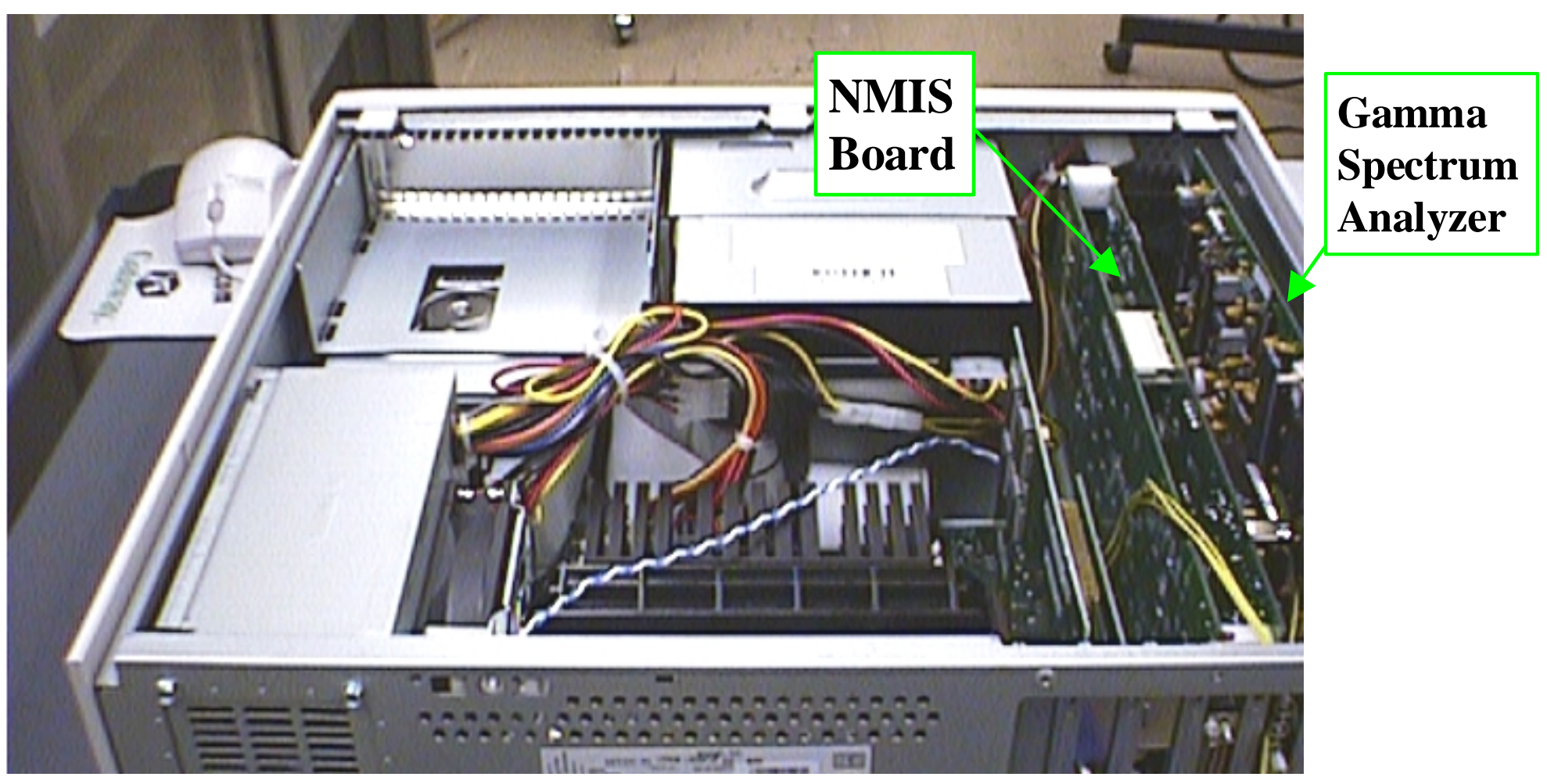

Fig. A-1. NMIS processor with gamma ray spectrum analyzer. 


\section{REFERENCES}

1. T. E. Valentine and J. T. Mihalczo, "MCNP-DSP: A Neutron and Gamma Ray Monte Carlo Calculation of Source-Driven Noise-Measured Parameters," Annals of Nuclear Energy 23, 16, 1271 (1996).

2. T. E. Valentine and J. T. Mihalczo, "Validation of the Monte Carlo Code MCNP-DSP," Annals of Nuclear Energy 24, No. 2, 79-98 (1996).

3. J. K. Mattingly, J. A. March-Leuba, J. T. Mihalczo, R. B. Perez, and T. E. Valentine, "Passive NMIS Measurements to Estimate Shape of Plutonium Assemblies," Y/LB-15,988, presentation, December 1998.

4. J. K. Mattingly, J. A. March-Leuba, J. T. Mihalczo, R. B. Perez, and T. E. Valentine, "Passive NMIS Measurements to Estimate Shape of Plutonium Assemblies," Y/LB-15,988, R1, Video, December 1998.

5. J. K. Mattingly, J. A. March-Leuba, J. T. Mihalczo, R. B. Perez, and L. G. Chiang, "Passive NMIS Measurements to Estimate the Shape Attribute of Plutonium Assemblies," Institute of Nuclear Materials Management Conference, Phoenix, Arizona, July 25-29, 1999. 